\title{
LA CUENCA EXPERIMENTAL DE IZAS, PIRINEO ARAGONÉS
}

B. ALVERA

Instituto Pirenáico de Ecología (CSIC). Avda. Rgto. Galicia s/n 22700 Jaca (Huesca)

C. electrónico: balvera@ipe.csic.es

\begin{abstract}
RESUMEN. En este articulo se describe la instrumentación de la cuenca experimental de Izas y algunos de los resultados obtenidos hasta la fecha. La cuenca de Izas es representativa de las condiciones ambientales de la alta montaña mediterránea (vertiente sur del Pirineo central), con una abundante innivación y temperaturas extremas.

ABSTRACT. In this paper the author describes the instrumentation and results of the experimental cathcment of Izas. The catchment of Izas, located in the Central Pyrennes, is representative of the enviromental characteristics of the bigh Mediterranean mountain, with abundant snow and extreme temperatures.
\end{abstract}

Palabras clave: Hidrología, Cuenca experimental, Transporte de sedimentos, Pirineos Key words: Hydrology, Experimental catchment, Sediment transport, Pyrennees

\section{Introducción}

Estando interesados en el estudio de algunos procesos a nivel de cuenca (ciclo hidrológico; ciclos biogeoquímicos de los principales nutrientes; exportación de sedimentos), se iniciaron los trabajos con muestreos puntuales más o menos regulares (de periodicidad quincenal), con los que se obtenían instantáneas del estado de tales procesos a lo largo de todo el año (caudal mediante un aforado químico y concentraciones con toma de muestras de agua y su posterior análisis en el laboratorio). Pero las condiciones meteorológicas influyen, de manera que se evitaba muestrear en momentos incómodos (lluvia, nevadas), con lo que los sucesos que se producen durante esas circunstancias climáticas adversas -breves en el desarrollo temporal de los procesos estudiados, pero de extraordinaria importancia para alguno (por no decir la mayoría) de ellos- no estaban bien representados. Por ejemplo, la exportación de sedimentos en suspensión, asociada a avenidas provocadas por lluvias torrenciales, que ocupan al cabo del año unas pocas horas (equivalentes a ca. $0.3 \%$ del tiempo total si fueran 24 horas al año), pueden concentrar el $90 \%$ de la descarga de tales sedimentos. Otras formas de exportación, que pueden ser en determinados ambientes las más importantes por su constancia a lo largo del año, como la salida de solutos (erosión química), están distribuidas más uniformemente a lo largo del año, pero de todas formas siguen dependiendo de la distribución de la 
escorrentía, y con el sistema de muestreos puntuales condicionados por la meteorología, es muy difícil tener datos de esos momentos.

De modo que se decidió elegir una pequeña cuenca para concentrar esfuerzos, dotándola de sistemas de muestreo continuo (aforo para la hidrología y estación meteorológica con muestreo automatizado permanente).

La cuenca debía reunir una serie de características. Nos interesaba que fuese indicativa del ambiente de la alta montaña mediterránea, representante de buena parte de las condiciones existentes en la vertiente surpirenaica, pero con alta innivación (la existencia de cubiertas temporales, de hasta seis meses, de nieve es una de las peculiaridades de esos ambientes...). También se requería un substrato impermeable que permitiera controlar las salidas del ciclo hidrológico con la escorrentía superficial, una zona con determinada altitud y libre de intervenciones humanas importantes, cercana a Jaca y con una accesibilidad no demasiado dificultosa (sobre todo en invierno).

El lugar elegido, la Cuenca Experimental de Izas, cumple con la mayoría de estas condiciones (Fig. 1, Foto 1). Probablemente la peor servida sea la de la accesibilidad, sobre todo para el material pesado (lo que ya hizo imprescindible desde el comienzo de la obra el uso de helicóptero), y la personal en invierno (hasta 6 horas, ida y vuelta, si no se domina la técnica del esquí de travesía).

La cuenca de Izas se extiende sobre 33 ha, entre 2060 y $2280 \mathrm{~m}$ de altitud, en la cabecera del río Escarra (tributario del Gállego por su margen derecha). Sus coordenadas geográficas son: $0^{\circ} 25^{\prime} \mathrm{W}$ y $42^{\circ} 44^{\prime} \mathrm{N}$. Está situada sobre la zona axil de los Pirineos, donde afloran terrenos paleozoicos (Carbonífero). Litológicamente predominan pelitas esquistosas, con intercalaciones de areniscas, pizarras, areniscas micáceas y pelitas arenosas, en alternancias monótonas de espesor decimétrico a métrico. La geomorfología fue determinada por procesos glaciares (típica de la cabecera de un valle glaciar, con lagos de sobreexcavación ya colmatados).

La vegetación zonal corresponde a una estepa de alta montaña, dominando hemicriptófitos cespitosos de crecimiento amacollado. Está cubierta por pasto de cobertura variable, aprovechado durante 3 o 4 meses estivales por ganado lanar.

\section{Instrumentación}

La instrumentación existente en la cuenca de Izás se describe a continuación.

\section{Hidrología y Transporte de sedimentos}

Aforo de presa con vertedera en forma de "V", de $120^{\circ}$ (Foto 2). Este sistema facilita la medida del caudal al limitar el muestreo a la altura del agua sobre el vértice de la " $\mathrm{V}$ " (no hace falta la velocidad). Con una fórmula, que tiene en cuenta que en el momento en que salta el agua la velocidad es nula, se transforman alturas en 1/s. Para la medida de la altura se han utilizado desde el principio sensores de presión, que miden la presión ejercida por la columna de agua sobre el sensor, situado debajo del vértice de la " $V$ " (bastante por debajo además, para evitar que se vea afectado por la congelación del agua superficial, que puede alcanzar hasta unos $20 \mathrm{~cm}$ de 


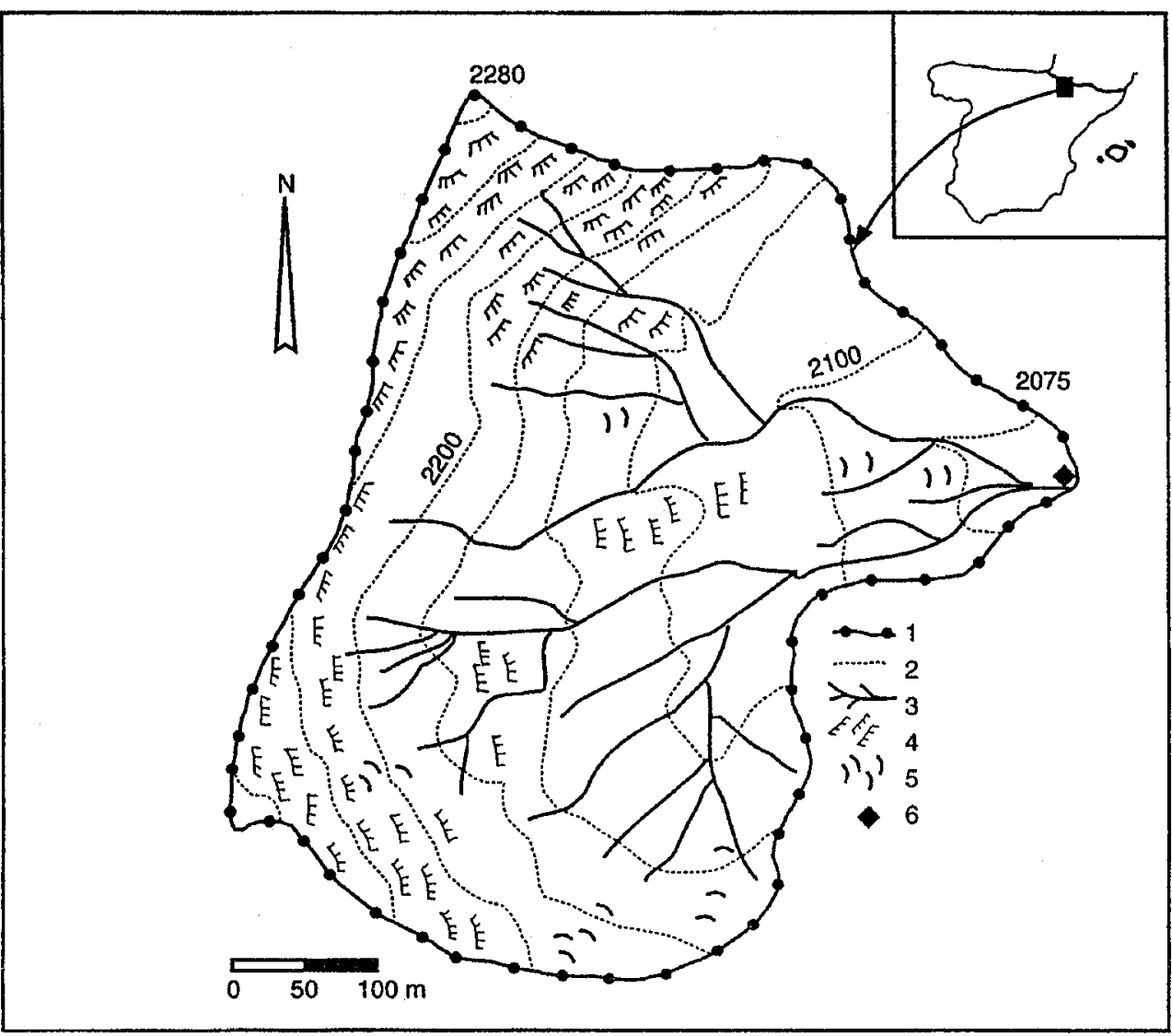

Figura 1. Cuenca experimental de Izas. Leyenda: 1) Divisoria principal; 2) Curvas de nivel; 3) Red fluvial; 4) Gradines o terracillas; 5) Lóbulos de solifluxión; 6) Presa de aforo y zona de instrumentación.

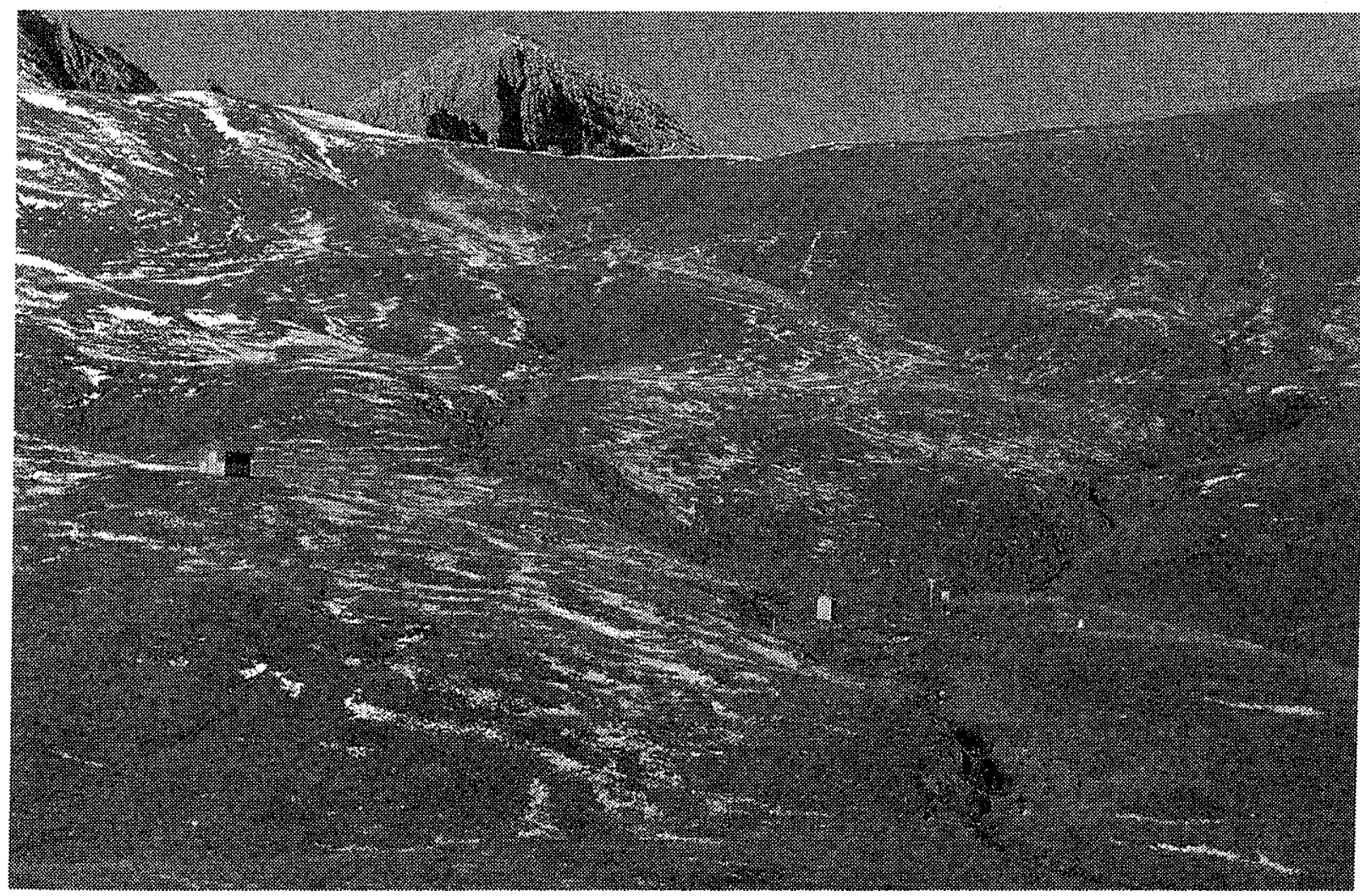

Foto 1. Vista general de la cuenca de Izas, con restos de nevada temprana (finales de septiembre de 2000). Refugio y zona de instrumentación. 
espesor en el embalse creado por la presa; este remanso es condición necesaria para que el agua al caer esté en reposo; el embalse tiene unos $15 \mathrm{~m}$ de longitud por un máximo de 5 de anchura). La altura de la columna de agua está relacionada linealmente con la presión ejercida por la misma. Se usaron sucesivamente hasta tres sensores piezorresistivos, que dieron muy mal resultado. A los pocos meses empezaban a dar lecturas erróneas, o a no responder adecuadamente a los cambios de presión/altura. El cuarto sensor (todavía en buen uso después de 6 años) es capacitivo (A.OTT ODS4P).

Recientemente (octubre de 1999) se ha instalado un sensor clásico de boya (Oche), que transmite una señal eléctrica también linealmente proporcional a la altura del agua. La verdad es que, funcionando bien el sensor de presión, no se han usado aún los datos del de boya. Presenta el inconveniente de que, cuando el agua superficial se congela, la boya queda temporalmente atascada a una altura, aunque el nivel del agua descienda después, mandando una señal constante. Cuando se descongela la lámina de hielo que la retiene, la boya cae bruscamente y se comienza a registrar un nivel inferior (muestreado adecuadamente, con una curva de descenso suave, por el sensor de presión).

Sensor de conductividad y temperatura del agua (Campbell 247). La principal forma de erosión en la cuenca es la química, a pesar de que el substrato es ácido y la concentración de sales en el agua es muy baja (pero se compensa por la continuidad de la escorrentía, que hace que constantemente estén saliendo solutos de la cuenca).

Para la concentración de sedimentos particulados se han utilizado 3 sensores de turbiedad. Los dos primeros no dieron muy buenos resultados. El tercero parecía

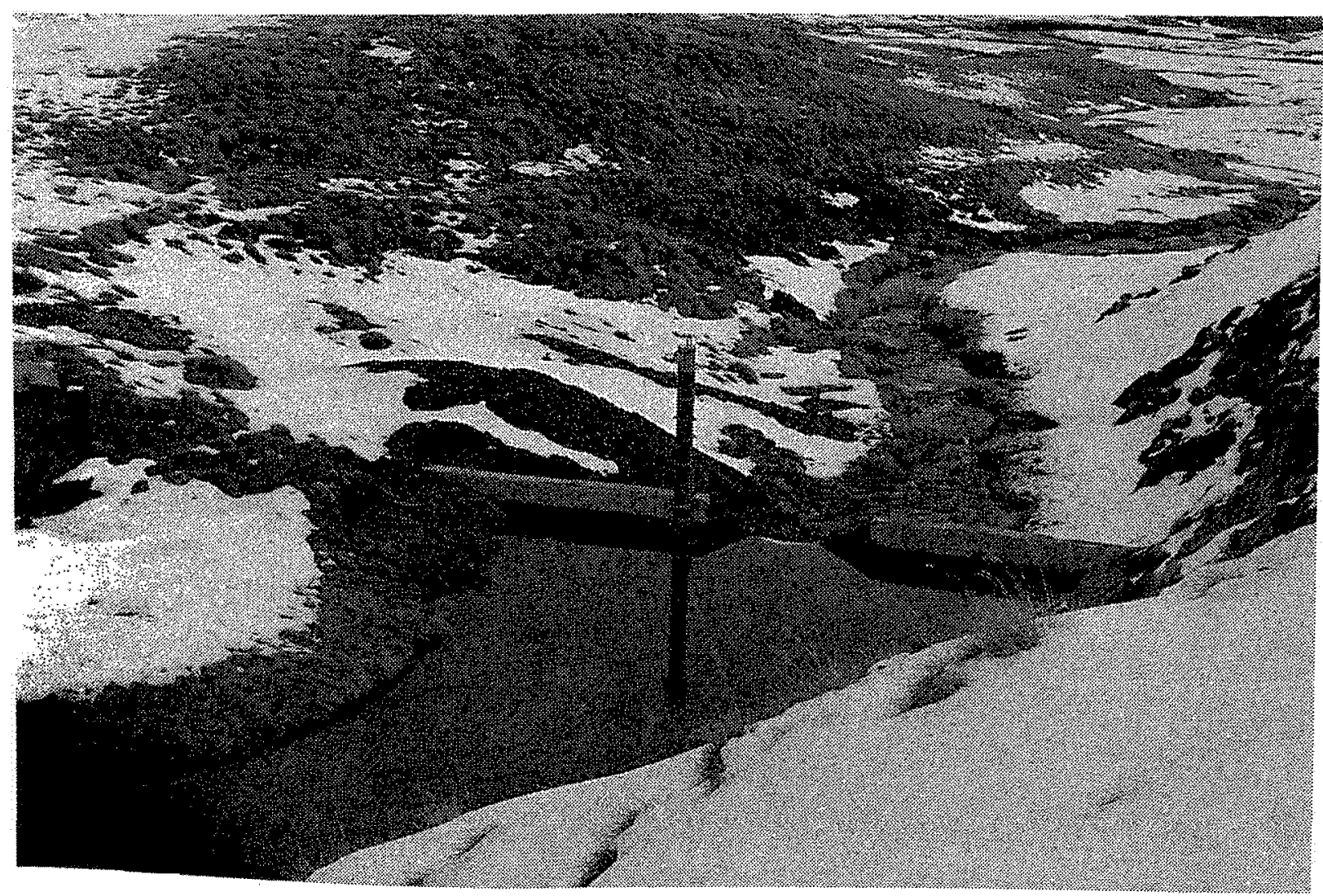

Foto 2. Presa con vertedera en $V$, tubo del limnígrafo de boya y embalse. 
bastante más fiable, pero se estropeó por congelación tras un corto período de funcionamiento y va a ser sustituido enseguida por uno nuevo de la misma marca y modelo (McVan Analite NEP195).

Los arrastres se miden por vaciado de una pequeña trampa de sedimentos situada sobre la cola del embalse. Esta resulta incapaz de retener los sedimentos cuando se producen importantes avenidas con arrastres, que se depositan entonces en la cola del embalse, de donde son retirados.

\section{Meteorología}

Precipitación. Es registrada por medio de un pluviógrafo con resolución de 0.1 $\mathrm{mm}$ (Weathemeasure 6011B; boca a $2.80 \mathrm{~m}$ del suelo, con escudo protector Alter).

Temperatura del aire y humedad. Actualmente están conectados 3 sensores (dos Vaisala HPM35A; el otro MCV SHR01). Están situados en una garita convencional del SMN, con la base a $1.80 \mathrm{~m}$ de altura sobre el nivel del suelo. En años de innivación relativamente importante (tal vez últimamente solo uno de cada cuatro o cinco años), el nivel de la nieve puede sobrepasar el de la garita o al menos el de su base. Esta circunstancia tiene dos efectos al menos: a) las condiciones en el interior de la garita, rodeada de nieve o justo por encima de ella, no representan las condiciones normales (debería de estar a 1.5-2 m sobre el suelo o la superficie); y b) el estar rodeada de nieve o tan cerca de ella favorece que la ventisca entre en la cabina y que se quede todo el interior ocupado por nieve, con lo que la temperatura y humedad que se miden en esas condiciones son las de la nieve y no las del aire. Esta circunstan-

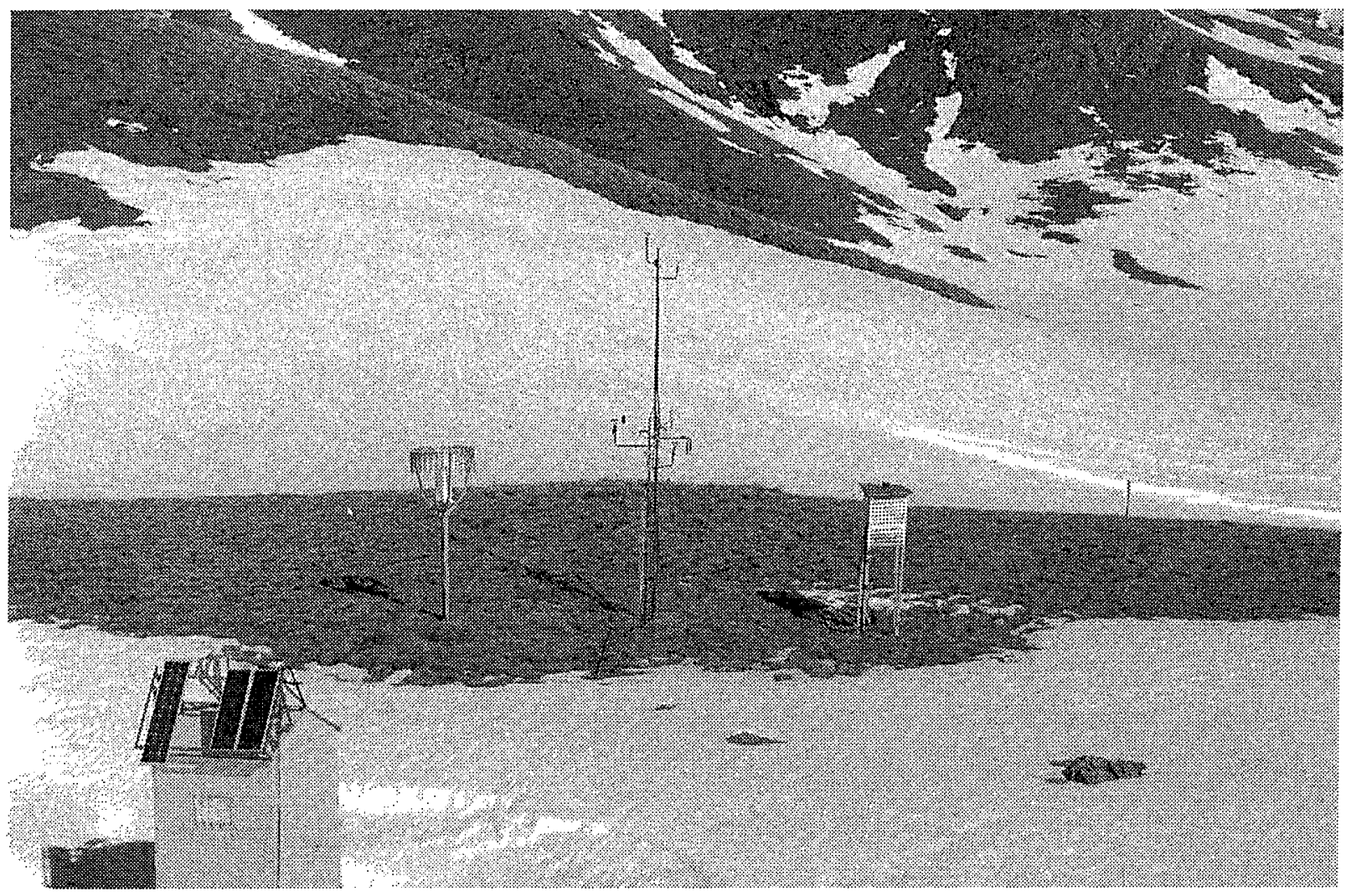

Foto 3. Zona de instrumentación: caseta del Sistemas de Adquisición de Datos, con placas solares, pluviografo, torre de sensores (radiómetros, anemómetros, etc), garita meteorológica. 
cia de ocupar la nieve el interior puede darse también aunque el nivel de aquella no sea muy alto y no esté tan próximo a la garita.

Para soslayar este problema se instaló un tercer sensor (Vaisala) a mayor altura sobre el suelo $-3.40 \mathrm{~m}$-, en un protector cilíndrico de láminas que no creemos que pueda ser alcanzado por la superficie de la nieve (el problema de la acumulación interior por ventisca puede seguir existiendo).

Como complemento de las medidas de temperatura y humedad se usan unos sistemas autónomos de sensores con registro incluido (Tinytalk II; sensores independientes para temperatura y humedad), con una capacidad de 1800 lecturas, ubicados en el interior de la garita meteorológica.

Viento. Dos sensores de velocidad (a 3.30 -Young 03002- y $6.25 \mathrm{~m}$-SEAC- de altura) y uno de dirección (3.30 m; Young 03002).

Espesor del manto de nieve. Ultrasónico, modelo UDG 01 de Campbell, funcionando durante 5 años; un nuevo modelo -SR50- de la misma marca desde diciembre de 2000 .

Radiación. Sensor de radiación global (piranómetro Campbell SP1110) situado sobre caseta de instrumentos, a unos $4 \mathrm{~m}$ del suelo; radiación solar incidente y reflejada (3.30 m; Swissteco SW2); radiación neta (pirradiómetro Thies 8111; $3.20 \mathrm{~m}$ ).

Temperatura del suelo. Perfil de siete profundidades, desde -5 a $-60 \mathrm{~cm}$; termistores Campbell 107.

Flujo de calor en el suelo. -2 y $-10 \mathrm{~cm}$ sin nieve; con nieve el superior se sitúa sobre el suelo, para medir el flujo entre la nieve y la superficie de aquel (REBS HTF-3.1).

Los Sistemas de Adquisición de Datos que funcionan actualmente son dos Datataker $(50$ y 500) y un Campbell CR10X (con un multiplicador de canales AM416). Los sensores se leen cada minuto y se guardan las medias (y en algunos casos, como el caudal, también las máximas) cada 15 minutos (de mayo a septiembre, aproximadamente) o cada hora (octubre-abril); en algunos sensores (dirección del viento, espesor de la nieve) se almacenan las lecturas instantáneas y no los promedios. Tres paneles solares alimentan una batería que proporciona la energía eléctrica necesaria para el funcionamiento de sensores y SAD.

Además, en verano se utilizan dos pluviómetros totalizadores y un termohigrógrafo de registro en papel, de duración semanal, además de termómetro de máximas y mínimas.

\section{El ambiente climático}

Para su descripción se recurre al último de los años hidrológicos completados (octubre 1999/septiembre 2000).

La temperatura media resultó ser de $5^{\circ} \mathrm{C}$ (extremas horarias: máxima, $21.6^{\circ} \mathrm{C}$; mínima, $-14.1^{\circ} \mathrm{C}$; extremos diarios: día más cálido, $18.4^{\circ} \mathrm{C}$ de media; día más frío, $12.5^{\circ} \mathrm{C}$; se registraron 60 dias con temperatura media negativa). La temperatura media del agua del torrente fue prácticamente igual a la del aire: $5.1^{\circ} \mathrm{C}$ (entre -0.1 y 24.3 de extremas horarias). La humedad relativa media fue de un $66 \%$. Se regis- 
traron $1842 \mathrm{~mm}$ de precipitación (distribuidos en 258 días, con un máximo diario de $119 \mathrm{~mm}$ y una distribución estacional del 52\% en primavera, 26 en otoño, 13 en verano y 9 en invierno). En 215 días se registró cubierta de nieve en el punto de medida, de los que en 107 el espesor fue mayor de $10 \mathrm{~cm}$; en las partes cóncavas alrededor de los cauces la duración de la cobertura nival es aún mayor, como un mes más).

La velocidad media del viento fue de $2.6 \mathrm{~m} / \mathrm{s}$, con un máximo horario de 13.4 . La dirección predominante del viento es del oeste, con un $38 \%$ de las observaciones horarias, seguido por el noroeste (17\%); entre ambos suman el 55\% de la frecuencia, repartiéndose los otros seis octantes el $45 \%$ restante (con porcentajes oscilando entre 5 y 10).

La radiación solar incidente media fue de $175 \mathrm{~W} / \mathrm{m}^{2}$. día (máximo diario de 379 y mínimo de $12 \mathrm{~W} / \mathrm{m}^{2}$ ); y la reflejada, $73 \mathrm{~W} / \mathrm{m}^{2}$ (con extremos diarios de 278 y 4 ). La cubierta nival influye especialmente en la radiación reflejada, que fue de media $104 \mathrm{~W} / \mathrm{m}^{2}$ con nieve y $46 \mathrm{sin}$, debido al mucho mayor albedo de la nieve que de la hierba (para la incidente también se notan diferencias: $135 \mathrm{~W} / \mathrm{m}^{2}$ con nieve y 209 sin; en este caso intervienen la mayor duración de los días a finales de primavera y verano y la mayor altura del sol sobre el horizonte).

El flujo de calor medio en el suelo a $-10 \mathrm{~cm}$ de profundidad (y entre el 11.11.99 y 30.09.00) fue de solo $0.68 \mathrm{~W} / \mathrm{m}^{2}$ (con máximo diario de 23 y mínimo de -23), diferenciándose la época cubierta de nieve $\left(-4.9 \mathrm{~W} / \mathrm{m}^{2}\right)$ de la $\sin (5.6)$. Bajo la nieve, el flujo entre ésta y la superficie del suelo fue de $-6.8 \mathrm{~W} / \mathrm{m}^{2}$.

\section{Resultados}

Intercambio de energía entre la atmósfera y el manto nival (Diez, 1991)

Para la temporada de fusión, se realizaron medidas de la radiación de onda corta (incidente y reflejada) y larga (incidente y emitida); por otra parte se calcularon esos valores con distintas aproximaciones dependientes de la posición del sol en el cielo (latitud geográfica, declinación solar, ángulo de la hora solar ) y para distintas condiciones de nubosidad (cielo despejado y cubierto), que permiten estimar muy exactamente la radiación. Luego se desarrolló un modelo distribuido de radiación potencial directa y difusa con el que se calcularon los valores para cada pixel del Modelo Digital de Elevaciones (MDE), teniendo en cuenta la autosombra dentro de la cuenca. Los cálculos se repitieron para los días 1 y 15 de cada mes del año.

\section{Variabilidad espacial de la nieve y su fusión (Anderton, 2000)}

La información obtenida mediante muestreos del espesor de nieve a lo largo de varios transectos longitudinales y transversales sugiere: a) que la distribución de la nieve al inicio del período de fusión depende de la influencia de la topografía en la redistribución de la nieve por el viento; y b) que el esquema espacial de fusión depende principalmente de la distribución del "equivalente hídrico de la nieve" al comienzo de la fusión, y no de la variabilidad espacial de las tasas de fusión. Se desa- 
rrolla un modelo distribuido de los procesos de fusión, que confirma lo sugerido por los muestreos para ambos extremos.

Distribución de ecosistemas (cartografía automática) (del Barrio et al., 1997; del Barrio, 1992, 1993)

La cuenca se cartografió a escala 1:1000, lo que produjo un mapa con curvas de nivel de una resolución de $1 \mathrm{~m}$. Digitalizando una de cada 5 curvas se elaboró un Modelo Digital de Elevaciones raster con pixels de $10 \mathrm{~m}$ de lado (posteriormente se realizó una nueva digitalización incluyendo todas las curvas, de la que se obtuvo un MDE con pixels de $1 \mathrm{~m}$ de lado, pero no se ha usado para el trabajo que se va a describir). Sobre esta base se realizaron (dentro del Sistema de Información Geográfica -SIG-Idrisi) mapas de pendientes y orientaciones. Además se utilizó un mapa de radiación solar directa en invierno (de un algoritmo desarrollado por C. Diez). Con TOPMODEL se calcularon otras dos capas hidrológicas: área drenada por unidad de curva de nivel e índice topográfico. Y un mapa de profundidad del substrato.

El estudio de la profundidad del substrato se obtuvo a partir del trabajo realizado por un equipo de edafólogos (dirigidos por A. Solé), que cartografiaron los suelos de la cuenca. El mapa de suelos también fue digitalizado y se reconocen cuatro tipos distintos: Leptosoles (que ocupan el $43 \%$ de la superficie), Cambisoles (46\%), Regosoles (4\%) y Gleysoles (6\%).

Por otra parte, del Barrio y Puigdefábregas hicieron un muestreo de vegetación y geoformas en los casi 200 puntos que habían sido muestreados por los edafólogos. Con los inventarios de vegetación (se detectaron 38 taxones de los cuales solo cuatro aparecieron en un cuarto o más de los puntos: Festuca eskia, 78\%, Nardus stricta, 77\%, Trifolium alpinum, 37\%, y Festuca rubra-nigrescens, 25\%) se llegó mediante TWINSPAN a una clasificación de seis clases. Las geoformas se consideraron como presencia/ausencia, con ocho categorías (lóbulos de suelo, terracillas o gradines cóncavos o convexos, formas de congelación superficial del suelo, canalizaciones subterráneas, escorrentía laminar, rastros de micromamíferos, indiferenciado).

Combinando mediante Análisis Discriminante Binario (ADB) los datos de suelos, vegetación y geoformas, se obtuvieron diez tipos de ecosistemas.

Por último, cruzando en el SIG los datos topográficos con los ecosistemas, se predijo la distribución de éstos en la cuenca. De los diez ecosistemas iniciales, ocho resultaron tener distribuciones significativas.

Este método de cartografía automática (y el de la elección del tamaño de pixel que se describe a continuación), interesaron a los creadores del SIG Idrisi, que los incluyeron como ejercicios prácticos en el $5^{\circ}$ volumen de una serie sobre aplicaciones de los SIG (Schneider, 1994 a y b).

Elección objetiva de tamaño de pixel (del Barrio et al., 1993)

Basándose en la teoría de la información, se desarrolló un método objetivo para elegir el tamaño de pixel a partir de las curvas de nivel digitalizadas. Se supone que la cantidad de información aumenta a medida que disminuye el tamaño del pixel, mientras las curvas añaden nueva información topográfica en cada paso, hasta alcan- 
zar un umbral crítico a partir del cual no hay incremento significante de información. Aplicado a las curvas digitalizadas de Izas, el tamaño óptimo de pixel que recoge el máximo de información sin redundancia resultó ser precisamente el que se había elegido intuitivamente como más adecuado $\left(10^{*} 10 \mathrm{~m}\right)$ y había sido utilizado en todas las pruebas.

Producción de gravas (su relación con la precipitación otoñal) (Alvera y GarcíaRuiz, 2000; García-Ruiz y Alvera, 1998; White et al., 1997)

Las gravas de la carga de fondo han precisado de más atención que las otras formas de exportación de sedimentos (solutos y sólidos en suspensión), pues mientras las segundas salen de la cuenca sin impedimento, las primeras quedan retenidas en la trampa colocada al efecto (cuando son pocas), o en la cola del pantano, haciendo obligatoria su remoción (de no haberse hecho, hace tiempo que el lago artificial creado por la presa estaría colmatado y sería inútil para medir el caudal). Unos 75 Mg (toneladas métricas) de gravas han tenido que ser sacados con palas y carretillas y arrojados aguas abajo de la presa (nunca se agradecerá lo bastante el esfuerzo realizado principalmente por una sola persona del Instituto, con algunas ayudas ocasionales, en esta tarea).

La producción de gravas se concentra principalmente en otoño, con ocasión de las lluvias torrenciales provocadas por las borrascas en esa estación, generadoras de grandes y rápidas escorrentías con energía suficiente para movilizar las gravas y transportarlas aguas abajo en intervalos de tiempo que deben ser extraordinariamente cortos (coincidiendo con los picos de las avenidas hidrológicas). En once años se han dado circunstancias suficientemente diferentes para encontrar que el volumen de gravas movidas en otoño depende principalmente de la intensidad de la lluvia en 24 horas consecutivas. Este es el intervalo de tiempo más corto que se ha considerado para relacionarlo con el total de gravas exportadas en otoño (octubre y noviembre), explicando hasta el 93\% (r2=0.93) de esa descarga (la intensidad de la lluvia en días naturales y la precipitación total en los dos meses llegaban a explicar el 88 y solo el 59\%, respectivamente, de la producción de gravas). E1 restante $7 \%$ sin explicar dependerá de algunos factores que parecen claros, como puede ser el tiempo transcurrido entre dos avenidas importantes, que si es muy corto no da tiempo a que se generen nuevas gravas en la inmediaciones de los cauces (con procesos como la gelifracción, o la acción de la gravedad sobre fragmentos ya desmenuzados) que puedan ser transportadas por la siguiente avenida

Se acaba de mencionar el otoño como época principal para el arrastre la carga de fondo, y así es efectivamente en los años en que se producen los máximos arrastres $(25,18,12.5 \mathrm{Mg}$ o toneladas métricas), y también en los intermedios (en siete de once casos así sucede; del $97 \%$ hasta menos del $2 \%$ puede ser la contribución otoñal). Cuando los arrastres son medios (entre 3 y $5 \mathrm{Mg}$ ) o bajos $(0.2$ a $0.5 \mathrm{Mg}$ ) puede suceder que sean las avenidas provocadas por las tormentas estivales las que movilicen la mayoría de las gravas (hasta el 95\% del escaso total anual, desde el 1\%). Incluso las suaves escorrentías primaverales, provocadas por la fusión del manto nival depositado en invierno y primavera, contribuyen al desalojo del 17\% de las gravas anuales en años de escasa producción. 
En primavera (en dos meses, el 12\% del tiempo) puede salir de la cuenca hasta alrededor del $70 \%$ de la escorrentía anual. Según este solo dato podría esperarse una mayor participación en la movilización de las gravas. Pero la fusión nival tiene unas características completamente distintas a la generación de escorrentía por la lluvia sobre el terreno despejado. La fusión se organiza día tras día, desde su inicio hacia mediados de abril o principios de mayo, en pulsos diarios, más o menos amplios dependiendo del contraste de temperaturas día/noche y la intensidad de la insolación, pero que ni en su apogeo alcanzan picos comparables a los debidos a las lluvias. Además los cauces permanecen cubiertos de nieve casi todo el tiempo (al comienzo de la fusión, toda la cuenca puede estar cubierta por nieve, pero la cobertura va desapareciendo progresivamente, comenzando por las zonas convexas -con espesores mínimos- de modo que las zonas cóncavas, precisamente las más cercanas a los cauces y en donde se encuentra la reserva de gravas susceptibles de ser arrastradas, son las que más tiempo mantienen la cobertura nival). Todo sucede suave y regularmente día tras día, comparado con los rápidos efectos de una lluvia torrencial.

Consecuentemente con la menor capacidad de la escorrentía invernal y primaveral para transportar gravas, resulta que la contribución de la fracción más gruesa de las mismas (clasificadas en 8 categorías entre $<0.5 \mathrm{y}>30 \mathrm{~mm}$ ) es mucho menor en esas condiciones que en verano y otoño. Más de la mitad del peso de gravas está formado por el tamaño entre 2 y $8 \mathrm{~mm}$; las fracciones menores (entre 2 y $<0.5 \mathrm{~mm}$ ) no pasan del $26 \%$ y las mayores $(>8 \mathrm{~mm}$ ) oscilan entre 20 y $44 \%$ (MartínezCastroviejo, 1990 a, b; Martínez-Castroviejo et al., 1991):

\author{
Grandes (>8 mm): 20 (primavera)-44\% (otoño) \\ Medias (2-8 mm): 45 (otoño)-65\% (primavera) \\ Pequeñas $(<2 \mathrm{~mm}$ ): 11 (otoño)-26\% (verano)
}

Exportación de sedimentos finos y solutos (Diez y Alvera, 1989; Diez et al., 1988, 1991)

La concentración de solutos (aniones y cationes: conductividad) en conjunto depende de la escorrentía: los caudales mínimos, de base, son los más concentrados al discurrir el agua lentamente a través del substrato cargándose de sales hasta acceder a los cauces (sequía estival y en invierno, con caudales mínimos bajo la nieve). Los incrementos de caudal provocados por la regular fusión nival primaveral o por las irregulares tormentas estivales y borrascas otoñales, diluyen los contenidos de solutos, pero en el balance anual esa reducción de concentración es mucho menor que la importancia del aumento del caudal, por lo que al final las salidas de solutos están gobernadas por la escorrentía, con una fase importante en primavera y otras distribuidas en verano y otoño.

Los sedimentos finos, aquellos que abandonan la cuenca suspendidos en el agua de escorrentía, dependen directamente del caudal. La mayor parte del tiempo el agua baja transparente (cargada de solutos, pero vacía de sedimentos sólidos), y sólo 
en algunos momentos (en total pueden sumar algunos días a lo largo del año) los caudales crecen lo suficiente como para poder poner en movimiento esta forma de erosión.

Por último, el arrastre de gravas de fondo exige energías tan grandes que solamente ocurren durante algunos minutos al cabo del año.

Como ejemplo pondremos las salidas de los tres componentes durante el año hidrológico 1998/99 (de octubre a septiembre), en $\mathrm{g} / \mathrm{m}^{2}$; y la precipitación (PR) y la escorrentía (Q) en $\mathrm{mm}$.

\begin{tabular}{|l|c|c|c|c|c|}
\hline Mes & PR & Q & Solutos & Sedimentos & Gravas \\
\hline Octubre & 89.4 & 131.7 & 13.8 & 1.2 & 0 \\
Noviembre & 100.4 & 54.0 & 7.1 & 0.2 & 0 \\
Diciembre & 106.9 & 15.2 & 5.9 & 0.5 & 0 \\
Enero & 98.2 & 15.2 & 2.4 & 0 & 0 \\
Febrero & 38.0 & 10.2 & 1.7 & 0 & 0 \\
Marzo & 260.7 & 42.7 & 6.0 & 0.1 & 0 \\
Abril & 258.3 & 194.3 & 20.9 & 2.3 & 0 \\
Mayo & 287.2 & 855.6 & 66.1 & 37.1 & 1.8 \\
Junio & 83.7 & 154.9 & 12.6 & 2.0 & 0 \\
Julio & 168.5 & 43.2 & 5.5 & 0.4 & 1.5 \\
Agosto & 113.5 & 66.8 & 8.5 & 0.4 & 0 \\
Septiembre & 448.3 & 369.2 & 34.7 & 20.5 & 11.8 \\
Total & 2053.1 & 1988.3 & 185 & 65 & 15 \\
Porcentaje & & & 70 & 25 & 5 \\
\hline
\end{tabular}

Durante el período reseñado, los principales aportes de solutos y sedimentos en suspensión se produjeron durante la fase culminante del deshielo, mayo, mes en el que se originó el 43\% de la escorrentía de la cuenca a lo largo de todo el año. Las tormentas de septiembre (con intensidades de hasta $150 \mathrm{~mm} /$ día, el $7 \%$ de lo caído en todo el año), que originaron el $22 \%$ de las precipitaciones anuales y propiciaron el $19 \%$ de la escorrentía anual, proporcionaron las intensidades puntuales de escorrentía que arrastraron el $79 \%$ de las gravas salidas de la cuenca (y los segundos puestos, tras mayo, para solutos y suspensión). Un otoño relativamente seco provocó la particularidad de que no se produjera ningún arrastre de fondo, cuando lo normal es que sea esta estación la que predomine en ese aspecto.

Las proporciones entre las tres formas de exportación, (70/25/5) se acercan bastante a lo registrado en años "normales" (entre aproximadamente 80/15/5 y $85 / 15 / 1)$. 
Para los nutrientes la situación más normal respecto al comportamiento de las concentraciones en relación con el caudal es la de que se produzcan diluciones con los incrementos. Esto sucede, por ejemplo, con el magnesio, sodio, sulfato, sílice, calcio, alcalinidad, en todas las condiciones (durante la fusión primaveral y en verano/otoño), y se debe a la procedencia de esos solutos, provenientes del lavado del substrato, -origen lítico-, que son diluidos del caudal de base por las aportaciones de agua superficial en fusión y lluvias.

Para el fósforo, de origen superficial (vegetación, mantillo, suelo superficial), los principales aportes se realizan cuando se produce escorrentía superficial que lava la superficie de la cuenca, con lo que se concentra, como los sedimentos finos, con los incrementos de caudal. Hay otros nutrientes cuya principal, pero no exclusiva, fuente también es superficial, como potasio, cloruro, nitrato. En estos casos el comportamiento no es homogéneo, respondiendo a los incrementos de escorrentía unas veces con aumentos y otras con diluciones en la concentración (Diez et al., 1991).

\section{Agradecimientos}

Distintos proyectos del CSIC, la CICYT, la DGA, contribuyeron al establecimiento de la cuenca. El mantenimiento de las instalaciones y la continuidad de la toma de datos se realiza en los últimos años gracias al programa RESEL (Red de Estaciones Experimentales de Seguimiento y Evaluación de la Erosión y la Desertificación ) del proyecto LUCDEME (Dirección General de Conservación de la Naturaleza). Emilio Ubieto es la persona del IPE que ha movido la mayor parte de la producción de gravas y ha materializado la instalación de muchos sensores.

\section{Bibliografia}

ALVERA, B. \& GARCÍA-RUIZ, J.M.,, 2000. Variability of sediment yield from a high mountain catchment, Central Spanish Pyrenees. Arctic, Antarctic, and Alpine Research,

ANDERTON, S., 2000. An analysis of spatial variability in snow processes in a bigh mountain catchment. Tesis doctoral. Universidad de Durham: $251 \mathrm{pp}$.

DEL BARRIO, G., 1992. Respuesta topográfica del paisaje en alta montaña: Pirineos Centrales. Tesis doctoral. Universidad Autónoma de Madrid: $160 \mathrm{pp}$.

DEL BARRIO, G., 1993. Respuesta topográfica del paisaje en alta montaña: Pirineos Centrales (resumen de tesis doctoral). Ecosistemas, 4: 52.

DEL BARRIO, G., ALVERA, B. \& DIEZ, J.C., 1993. The choice of cell size in Digital Terrain Models: an objective method. In Methods of Hydrological Basin Comparison (M. Robinson, ed.). Institute of Hydrology Rep. No. 120: 190-196.

DEL BARRIO, G., ALVERA, B., PUIGDEFÁBREGAS, J. \& DIEZ, J.C., 1997. Response of high mountain landscape to topographic variables: Central Pyrenees. Landscape Ecology, 12 (2): 95-115.

DÍEZ, J.C., 1991. Estudio y parametrización del intercambio de energía entre la atmósfera y el manto nival durante el período de fusión: Cuenca Experimental de Izas, Pirineos Centrales. Tesis doctoral. Universidad de Zaragoza: 167 pp. 
DÍEZ, J.C. \& ALVERA, B. 1989. Sólidos suspendidos y disueltos en la escorrentía de una pequeña cuenca pirenaica. Options Mediterranéennes, Série A; $\mathrm{n}^{0} 3:$ 237-241.

DÍEZ, J.C., ALVERA, B., DEL BARRIO, G. \& PUIGDEFÁBREGAS, J., 1991. Streamflow-water chemistry relationships in the alpine environmen: a case study in a Pyrenean watershed. Mitteilungsblatt des Hydrographischen Dienstes in Österreich, 65/66: 119-123.

DÍEZ, J.C., ALVERA, B., PUIGDEFÁBREGAS, J. \& GALLART, F., 1988. Assessing sediment sources in a small drainage basin above timberline in the Pyrenees. Sediment Budgets. IAHS Publ. $\mathrm{n}^{\circ}$ 174: 197-205.

GARCÍA-RUIZ, J.M. \& ALVERA, B., 1998. La exportación de sedimentos en alta montaña. El ejemplo de la cuenca experimental de Izas, valle del Gállego, Pirineo Aragonés. In: Procesos biofísicos actuales en medios fríos (Gómez-Ortiz et al., eds.). Univ. Barcelona: 235244.

MARTÍNEZ-CASTROVIEJO, R., 1990a. Bed load size distribution and flow conditions in a high mountain catchment of Central Pyrenees. Pirineos, 135: 39-54.

MARTÍNEZ-CASTROVIEJO, R., 1990b. La exportación de sedimentos gruesos en relación con el régimen bidrológico en una cuenca de alta montaña (Cuenca de Izas, Alto Gállego, Pirineo aragonés). Tesis de licenciatura. Universidad de Zaragoza: 152 pp.

MARTÍNEZ-CASTROVIEJO, R., GARCÍA-RUIZ, J.M., DIEZ, J.C. \& ALVERA, B., 1991. Coarse sediment transport in an experimental high mountain catchment of Central Pyrenees, Spain. Z. Geomorph. N.F. Suppl.-Bd. 83: 105-114.

SCHNEIDER, K. (ed.), 1994a. United Nations Institute for Training and Research (UNITAR). Explorations in Geographic Information Systems Technology. Vol. 5: GIS and Mountain Environments. Exercise 8: Spatial modelling of significant relationships between ecosystems and topography: págs. 8-1 a 8-15.

SCHNEIDER, K. (ed.), 1994b. United Nations Institute for Training and Research (UNITAR). Explorations in Geographic Information Systems Technology. Vol. 5: GIS and Mountain Environments. Exercise 1: Choosing pixel resolution: An objective method: págs. 1-10 a 1-12. Appendix IV: An objective method for determining cell resolution: págs. IV-1 y IV-2.

WHITE, S., GARCÍA-RUIZ, J.M., MARTÍ, C., ALVERA, B. \& DEL BARRIO, G., 1997. Sediment transport in a high mountain catchment in the Central Spanish Pyrenees. Phys. Chem. Earth, 22 (3-4): 377-380. 